\title{
Effect of acceptor and donor dopants on polarization components of lead zirconate titanate thin films
}

\author{
S. B. Majumder, B. Roy, and R. S. Katiyar ${ }^{\text {a) }}$ \\ Department of Physics, University of Puerto Rico, San Juan, Puerto Rico 00931-3343
}

S. B. Krupanidhi

Materials Research Center, Indian Institute of Science, Bangalore, 560012, India

\begin{abstract}
We have compared the magnitudes of reversible and irreversible polarization components of solgel-derived $\mathrm{Nd}^{3+}$ - and $\mathrm{Fe}^{3+}$-doped PZT (53/47) thin films on platinized silicon substrates. Beyond the switching field, it was found that the reversible component of the polarization remains almost constant both for donor- $\left(\mathrm{Nd}^{3+}\right)$ and acceptor- $\left(\mathrm{Fe}^{3+}\right)$ doped PZT films. The irreversible polarization component reduces with the increase in $\mathrm{Nd}^{3+}$ content, whereas it increases until 3 at. \% $\mathrm{Fe}^{3+}$-doped PZT thin films. The dielectric behavior of these films at subswitching fields was analyzed in terms of Rayleigh law. The inverse of the Raleigh coefficient $(v)$ was considered as a measure of the obstacle for the domain-wall motion. In the case of Fe-doped PZT, the inverse of the Raleigh coefficient $(v)$ shows a declining linearity with Fe content, which may be found exactly opposite to that observed for Nd-doped PZT. The observed results are explained in terms of the nature of the defect-domain-wall interaction of acceptor-and-donor-doped PZT thin films. Studies indicated that in the case of Fe-doped PZT films, the possible defect interaction initiates only above 2 at. \% and it was found to be much lower in magnitude in comparison with the $\mathrm{Nd}$ doping.
\end{abstract}

Defect distributions and their subsequent mobility has a direct impact on the polarization switching, domain morphology, and ferroelectric phase purity of PZT thin films for ferroelectric random access memory applications. ${ }^{1-3}$ The mobile defects are believed to be dipolar and can establish a strong interaction with the domain walls. Such coupling can lead to a possible domain-wall pinning, while this process is further influenced by extrinsic cation additions as dopants. The donor dopant, such as $\mathrm{Nd}^{3+}$, induces cationic defects while occupying the $A$ site ${ }^{4,5}$ of the $\mathrm{ABO}_{3}$ perovskite-like lattice. On the other hand, the acceptor-type dopants enhance oxygen vacancies while occupying the $B$ sites. ${ }^{6}$ Such differential behavior may impart varied effects on the polarization behavior through interaction with the domain walls. ${ }^{7,8}$

The present letter deals with the theme of understanding the effect of the nature of doping on the reversible and irreversible polarization components in PZT films with $\mathrm{Nd}$ as the donor dopant occupying the $A$ site, and Fe as the acceptor dopant to occupy the $B$ site. A comparative study was conducted in terms of their influence on the reversible and irreversible polarization components.

We have synthesized $\mathrm{Pb}_{1.0-3 x / 2} \mathrm{Nd}_{x}\left(\mathrm{Zr}_{0.53} \mathrm{Ti}_{0.47}\right) \mathrm{O}_{3}$ $\left(x=0-10\right.$ at. \%) and $\mathrm{Pb}\left(\mathrm{Zr}_{0.53} \mathrm{Ti}_{0.47}\right)_{1-x} \mathrm{Fe}_{x} \mathrm{O}_{3-\delta} \quad(x=0-5$ at. \%) thin films on platinized silicon substrates by the solgel technique. Just after deposition the films were inserted in a preheated furnace at $600{ }^{\circ} \mathrm{C}$ and fired for 5 min to remove the organics. The coating and firing cycle was repeated to achieve films of about $0.4-0.5 \mu \mathrm{m}$ thickness and they were finally annealed at $700{ }^{\circ} \mathrm{C}$ for $1 \mathrm{~h}$ in oxygen for crystalliza-

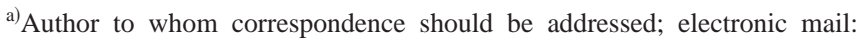
rkatiyar@rrpac.upr.clu.edu
}

tion into the perovskite phase. The phase formation behavior was studied using x-ray diffraction (XRD). For the top contact, Au electrodes of $0.05 \mathrm{~cm}$ diam were deposited by $\mathrm{dc}$ sputtering, and a platinized silicon substrate was used as the bottom contact. The dielectric characteristics of the films were measured by an impedance analyzer (HP 4294A) and the ferroelectric characteristics were measured by a commercial hysteresis loop tester (6000 HVS, Radiant Technology).

With increasing Nd content, the remnant polarization decreased from $30 \mu \mathrm{C} / \mathrm{cm}^{2}$ (undoped) to $5 \mu \mathrm{C} / \mathrm{cm}^{2}$ (10 at. \% $\mathrm{Nd}$ doped), while the hysteresis loops became either unsaturated or slim. The polarization reduces moderately up to 4 at. \% $\mathrm{Nd}$ content and beyond that the reduction is abrupt. No significant effect is observed on the coercive field. The lattice parameters calculated from the x-ray diffractograms were found to be reduced monotonically with the increase in $\mathrm{Nd}$ content. Polarization in the ferroelectric perovskite lattice is contributed to by the displacement of higher valence $B$-site cations along the polar axis from their position in the paraelectric phase. ${ }^{9}$ The contraction of the lattice parameter is probably accompanied by a decrease in the rattling space available for $B$-site cations and, therefore, the polarization decreases moderately up to 4 at. $\% \mathrm{Nd}$ doping. The $\mathrm{x}$-ray analysis also revealed the coexistence of a pyrochlore phase beyond 4 at. \% $\mathrm{Nd}$, which can deteriorate the polarization abruptly. In contrast to that observed for $\mathrm{Nd}$ doping, the polarization values of $\mathrm{Fe}$-doped films are enhanced for up to 3 at. \% Fe doping, and reduce moderately beyond that. No pyrochlore phase formation was detected up to 5 at. \% Fe doping. The increase in polarization values could be due to enhanced crystallinity as indicated by the XRD data. The polarization behavior of the ferroelectric material was separated into reversible and irreversible components. ${ }^{10,11}$ The 


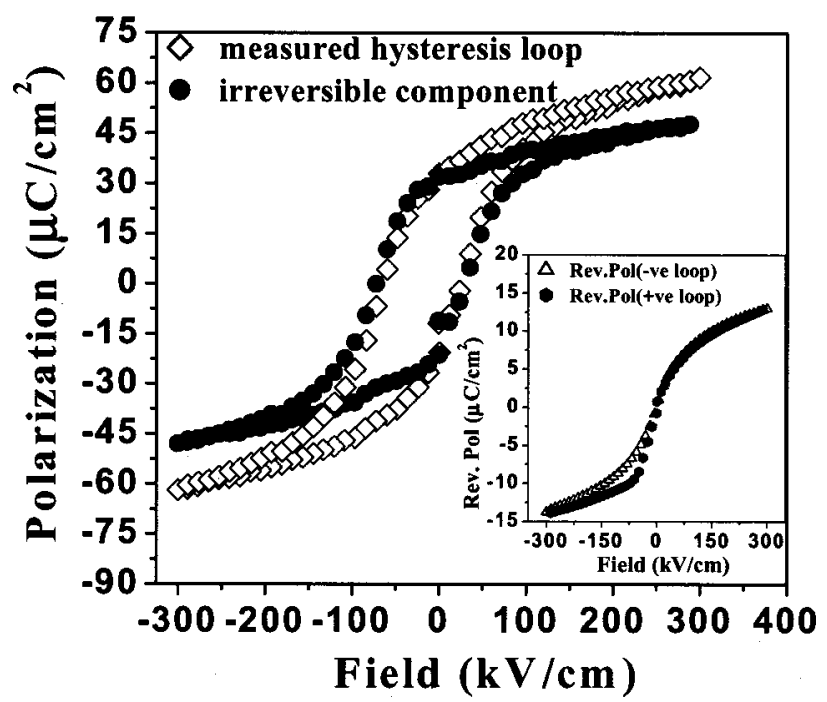

FIG. 1. As measured and irreversible component of polarization hysteresis of the undoped PZT thin film. The inset shows the reversible component of the polarization hysteresis measured from the $C V$ loop.

resolved irreversible component of polarization is shown in Fig. 1. The Fig. 1 inset shows the reversible polarization component calculated from the measured CV loop.

The values of reversible and irreversible polarizations at $V_{\max }$ (voltage corresponding to $P_{\max }$ of the hysteresis loop) as a function of (a) $\mathrm{Nd}$ and (b) Fe atomic contents are shown in Figs. 2(a) and 2(b), respectively. For Nd-doped PZT films the irreversible component decreased progressively with $\mathrm{Nd}$ content, and the reversible component remained almost stable at all dopant levels. Since $\mathrm{Nd}$ doping is expected to increase the defect concentration either at the $A$ or $B$ site of the $\mathrm{ABO}_{3}$ lattice, ${ }^{4,5}$ one could expect a direct interaction of these defects with the domain walls. ${ }^{7}$ In our study, the $\mathrm{Nd}$ content was increased up to 10 at. \%, which can offer two-
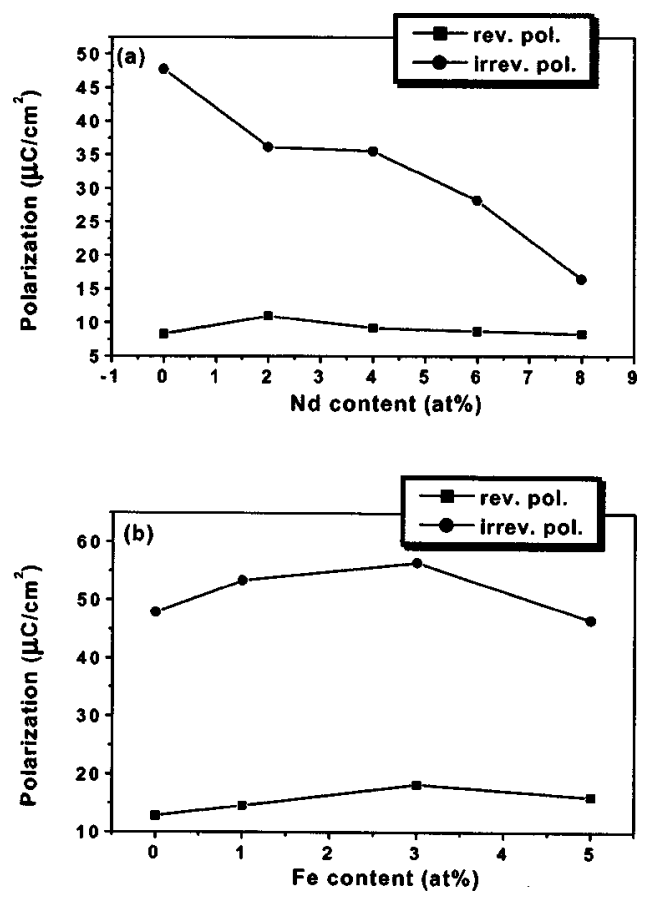

FIG. 2. Variation of the reversible and irreversible polarization component of (a) Nd-doped and (b) Fe-doped PZT thin films.
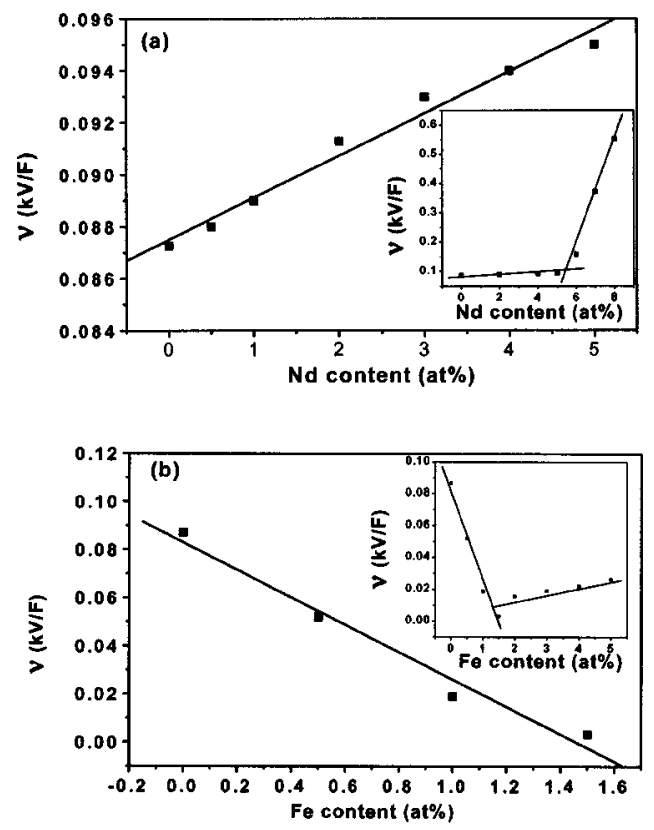

FIG. 3. Reciprocal Rayleigh constant $(v)(v=1 / \alpha)$ as a function of (a) $\mathrm{Nd}$ and (b) Fe dopant content.

fold reasons for the reduction of the irreversible component, such as: (a) The vacancy concentration of $\mathrm{Pb}$ would increase with the increase in $\mathrm{Nd}$ content, and thus higher defect concentrations occur at higher $\mathrm{Nd}$ doping levels, and these can interact with the domain boundaries, thus reducing the irreversible polarization component. (b) The reduction of the perovskite phase content in PZT films at doping levels beyond 4 at. \% (as envisaged from the XRD data) may also contribute to the observed reduction in the irreversible polarization component.

The irreversible polarization components remained at a higher value with a minor variation in the Fe-doped (up to 5 at. \% Fe) PZT thin films. The reversible component remained almost constant, similar to that observed in the case of $\mathrm{Nd}$ doped PZT films [Fig. 2(b)]. Fe doping is expected to induce oxygen vacancies in the PZT lattice. ${ }^{6,12}$ Evaporation of $\mathrm{PbO}$ during high-temperature annealing also induces both oxygen and lead vacancies in the lattice. Electron paramagnetic resonance studies have shown that defect dipoles such as $\mathrm{Fe}^{3+}-V_{\ddot{o}}$ complexes or $V_{\mathrm{Pb}}-V_{\ddot{o}}$ complexes exist in perovskite oxide. ${ }^{13}$ It is possible that in our case $\mathrm{Fe}^{3+}$ can bind oxygen vacancies in the unit cells containing substitutional Fe. ${ }^{14}$ Therefore, despite the fact that acceptor doping such as Fe introduces oxygen vacancies in the PZT lattice, it is possible that due to the formation of defect dipoles these defects do not affect the domain-wall distance or the interaction significantly.

Under the subswitching fields, the domain-wall contribution to the dielectric permittivity can be analyzed by the Rayleigh law originally applied to the ferromagnetic, ${ }^{15,16}$ and more recently, to ferroelectric materials. ${ }^{17}$ Similar to ferromagnets, ferroelectric polarization at subswitching fields $(E$ $<E_{C}$, where $E_{c}$ is the coercive field), can be expressed as

$$
\epsilon(E)=\epsilon_{0} \epsilon_{\text {init }}+\epsilon_{0} \alpha E,
$$

where $\epsilon$ is the permittivity, $\epsilon_{0}$ is the permittivity of free space, $\epsilon_{\text {init }}$ is the permittivity when no field is applied, $\alpha$ is 
the Rayleigh constant, and $E$ is the applied ac field. For a wide frequency range $(1 \mathrm{kHz}-1 \mathrm{MHz})$, the dielectric permittivity $(\epsilon)$ versus ac field $(E)$ data for undoped and Nd- and Fe-doped thin films could all be fitted to a straight line, as predicted in Eq. (1). According to the theory of Boser, the inverse of the Rayleigh constant (defined as $v$ ) is proportional to the concentration of defects (and thereby to the dopant concentration). ${ }^{18}$ Figures 3(a) and 3(b) show the variation of the reciprocal Rayleigh constant $(v)$ with the doping concentrations for $\mathrm{Nd}$ and $\mathrm{Fe}$, respectively. For Nddoped PZT films, $v$ holds the linear relationship up to 5 at. $\%$ and there is a steep increase in $v$ at higher dopant concentrations. Considering $v$ as a measure of obstacles for domainwall motion, the $\mathrm{Nd}$ dopant appears to interact directly with the domain-wall motion. The presence of phase-related inhomogenieties at higher $\mathrm{Nd}$ content may further hinder the domain-wall motion. After an increase of $\mathrm{Fe}$ beyond 1.5 at. $\%, v$ exhibited a linear increase with dopant concentration. These comparative observations indicate that the $\mathrm{Nd}$ dopant even at lower concentrations initiates a blocking of domain-wall motion, as it readily induces cationic (either $A$ or $B$ sites) defect concentration. However, in the case of Fe-doped PZT films, particularly at lower dopant concentration, the interactions between the defects and domain walls are minimized due to the formation of defect dipoles such as $\mathrm{Fe}^{3+}-V_{\ddot{o}}$ complexes or $V_{\mathrm{Pb}}-V_{\ddot{o}}$ complexes. Any possible defect interaction would start only above 1.5 at. \%, and was still found to be much lower in magnitude in comparison with Nd-doped PZT.

In the present work the reversible and irreversible polarization contributions of donor- $\left(\mathrm{Nd}^{3+}\right)$ and acceptor- $\left(\mathrm{Fe}^{3+}\right)$ doped $\mathrm{Pb}\left(\mathrm{Zr}_{0.53} \mathrm{Ti}_{0.47}\right) \mathrm{O}_{3}$ thin films have been compared. At $V_{\max }$, the irreversible polarization component decreased progressively with the increase in $\mathrm{Nd}$ contents, whereas in the case of the Fe-doped PZT, the irreversible component remained at a higher value with a small variation. The reversible and irreversible components of the dielectric permittivity have also been evaluated at the subcoercive field using Rayleigh law. Considering the inverse of the Raleigh coefficient $(v)$ as a measure of obstacles for domain-wall motion, it was found that it increases linearly up to 5 at. \% $\mathrm{Nd}$ doping and beyond that with a higher slope. It indicates that there is a strong interaction between the induced defects and the domain wall for up to 5 at. $\% \mathrm{Nd}$ content, and beyond that the phase separation and defect distribution have a combined influence towards the increase of the slope. In contrast, in the case for Fe-doped PZT, $v$ shows a declining linearity with Fe content, which may be found to be exactly opposite that for Nd-doped PZT. However, beyond 1.5 at. \% Fe content, $v$ exhibited a linear increase with dopant concentration. It indicates that in the case of Fe-doped PZT films any possible defect interaction starts only above 1.5 at. $\%$ and it was found to be much lower in magnitude in comparison with the Nd-doped PZT.

The work was supported by NSF-DMR Grant No. 9801759, DAAG Grant No. 55-98-1-0012, and NASANCC5-518 grants.

${ }^{1}$ Q. Tan, J. Li, and D. Viehland, Appl. Phys. Lett. 75, 418 (1999).

${ }^{2}$ Q. Tan, Z. Xu, and D. Viehland, J. Mater. Res. 14, 465 (1999).

${ }^{3}$ R. D. Klissurska, K. G. Brooks, I. M. Reaney, C. Pawlaczyk, M. Kosec, and N. Setter, J. Am. Ceram. Soc. 78, 1513 (1995).

${ }^{4}$ A. Garg and T. C. Goel, Mater. Sci. Eng., B 60, 128 (1999).

${ }^{5}$ S. R. Shanigrahi, R. N. P. Choudhary, and H. N. Acharya, Mater. Sci. Eng., B 56, 31 (1999).

${ }^{6}$ B. G. Chae, S. J. Lee, Y. S. Yang, S. H. Kim, and M. S. Jang, Jpn. J. Appl. Phys., Part 1 36, 7275 (1997).

${ }^{7}$ D. Bolten, U. Böttger, T. Schneller, M. Grossmann, O. Lohse, and R. Waser, Appl. Phys. Lett. 77, 3830 (2000).

${ }^{8}$ S. Takahashi, Ferroelectrics 41, 143 (1982).

${ }^{9}$ S. C. Abrahams, S. K. Kurtz, and P. B. Jamieson, Phys. Rev. 172, 551 (1968).

${ }^{10}$ D. V. Taylor and D. Damjanovic, J. Appl. Phys. 82, 1973 (1997).

${ }^{11}$ D. Bolten, O. Lohse, M. Grossmann, and R. Waser, Ferroelectrics 221, 251 (1999).

${ }^{12}$ R. D. Klissurska, K. G. Brooks, and N. Setter, Ferroelectrics 225, 171 (1999).

${ }^{13}$ W. L. Warren, G. E. Pike, K. Vanheusden, D. Dimos, B. A. Tuttle, and J. Robertson, J. Appl. Phys. 79, 9250 (1996).

${ }^{14}$ R. Lohkamper, H. Neumann, and G. Arlt, J. Appl. Phys. 68, 4220 (1990).

${ }^{15}$ L. Rayleigh, Philos. Mag. 23, 225 (1887).

${ }^{16}$ D. Jiles, Introduction to Magnetism and Magnetic Materials (Chapman and Hall, London, 1991), pp. 85-175.

${ }^{17}$ D. Damjanovic, Rep. Prog. Phys. 61, 1267 (1998); A. Fouskova, J. Phys. Soc. Jpn. 27, 1699 (1969).

${ }^{18}$ D. Boser, J. Appl. Phys. 62, 1344 (1987). 